\title{
Multi-Nuclear Relaxation Time Studies in Undercooled Aqueous Electrolytes
}

E. W. Lang, W. Fink, H. Radkowitsch, and D. Girlich

Institut für Biophysik und Physikalische Biochemie, Universität Regensburg, POB 397, D-8400 Regensburg, FRG

Diffusion / High Pressure / Liquids / Spectroscopy, Nuclear Magnetic Resonance / Transport Properties

The dynamic properties of water molecules coordinated to simple ions (alkali-, alkali earth halides) in undercooled solutions have been investigated with NMR. Relaxation time studies at low temperatures provide details of molecular motions as well as certain features of the local arrangement in the coordination sphere of these ions. 


\section{Introduction}

NMR relaxation rate studies of various nuclei are well suited to study the rotational and translational diffusion of water molecules and ions in electrolyte solutions. In undercooled solutions the relaxation rate curves $R_{1}(T, c, p)$ become sensitive to details of the spectral density of diffusive modes as well as local structural characteristics in the solutions.

At low temperatures a crystalline phase nucleates in metastable water due to the rapid completion of the $\mathrm{H}$-bond network. Ionic solutes and/or hydrostatic pressure act as network breaking agents and allow a sufficient degree of undercooling to be obtained [1]. Highly concentrated solutions may even form glasses readily [2].

In neat water molecular motions are isotropic. Dissolved ions disturb the local structure of the H-bond network and induce some anisotropy in the orientational fluctuations of coordinated water molecules [3]. A simple motional model [4] for these reorientational modes is considered which is consistent with the average local structure of the hydration sphere known from neutron scattering $[5,6]$ and computer simulations [7]. Together with a two-site approximation the model is used to interpret relaxation rate curves $R_{1}(T, c, \omega, p)$ of various nuclei in metastable solutions. Characteristics of cooperative structural fluctuations in undercooled liquids like a non-Arrhenius temperature dependence of transport coefficients and non-Debye spectral densities are discussed also.

\section{Hydration Water Dynamics}

\subsection{A Motional Model}

In aqueous solutions molecular motions occur on two largely separated time scales. Hindered translations and rotations can be considered as thermal excitations of the quasistatic $\mathrm{H}$-bond network $[8,9]$. At high temperatures these quasi-lattice vibrations (QLV) are strongly damped. At lower temperatures they are much faster than translational and rotational diffusive motions. Because of timescale separation QLV and diffusive motions can be considered to be statistically independent.

Neutron [5] and X-ray [6] scattering as well as computer simulations [7] provide details of the average local structure of the coordination sphere of ions. Water molecules always orient their hydrogen atoms away from cations, their dipole moment vector being tilted relative to the radial direction. Towards anions water molecules orient one of the $\mathrm{OH}$ bonds in an almost linear fashion. The width of the peaks in the corresponding radial distribution functions indicate the range of angles and distances allowed by the given interaction potentials.

Consistent with this average ion-water arrangement reorientational fluctuations of hydration waters are decomposed as follows $[4,9]$. The molecules execute small amplitude librations about their mean orientation. These fast torsional oscillations are superimposed onto anisotropic fluctuations around the local director (ion-oxygen distance) with correlation time $\tau_{\mathrm{i}}$. In addition the molecules tumble around the ion with correlation time $\tau_{\mathrm{r}}$ or leave the coordination sphere after a mean residence time $\tau_{\mathrm{ex}}$. Both modes are highly cooperative and will be combined to an effective tumbling mode $1 / \tau_{0}=1 / \tau_{\mathrm{r}}+1 / \tau_{\mathrm{ex}}$.

\subsection{Temperature Dependence of the Correlation Times}

In undercooled liquids cooperative motions slow down strongly causing, in fragile liquids [10], a characteristic nonArrhenius temperature dependence of transport and relaxation coefficients. This behaviour is well represented by the empirical VTF-equation [11]

$\tau_{0}=\tau_{00} \exp \left(B /\left(T-T_{0}\right)\right)$

It predicts a slowing down of cooperative molecular motions at the ideal glass transition $T_{0}$. The local anisotropic reorientation of the water molecules around the local director
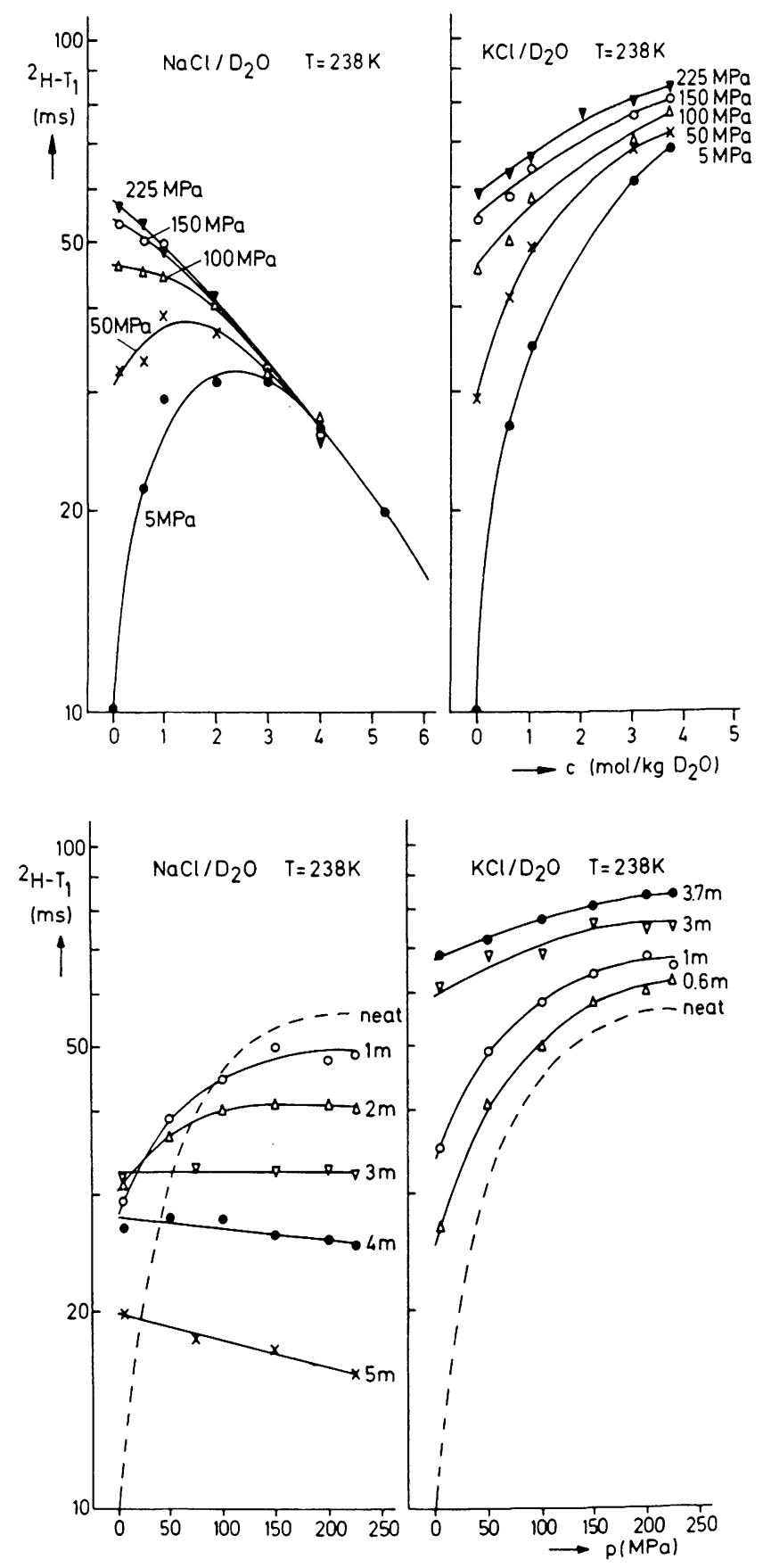

Fig. 1

Comparison of the effect of pressure and/or ionic solutes on the average rotational mobility $\left(\sim T_{1}\left({ }^{2} \mathrm{H}\right)\right)$ of water molecules in metastable solutions of $\mathrm{NaCl}$ and $\mathrm{KCl}$ in $\mathrm{D}_{2} \mathrm{O}$ 
can be considered a thermally activated mode with a characteristic Arrhenius behaviour

$\tau_{\mathrm{i}}=\left\langle 1 / v_{\mathrm{lib}}\right\rangle \cdot \exp \left(E_{\mathrm{a}} / k T\right)=\tau_{\mathrm{i} 0} \cdot \exp \left(E_{\mathrm{a}} / k T\right)$.

The preexponential factor corresponds to an attempt frequency for barrier crossing, hence is given by an average librational frequency of hydration water molecules [12].

\section{Multi-Nuclear Relaxation Experiments}

\subsection{Orientational Fluctuations of Water Molecules in the Hydrogen Bond Network}

Orientational fluctuations of water molecules in various metastable electrolyte solutions have been investigated through deuterium spin-lattice relaxation rates $R_{1}\left({ }^{2} \mathrm{H}\right)$ $[4,9,13-16]$. In the fast motions regime $(\omega \tau \ll 1)$ these rates reflect an average rotational mobility of water molecules only.

At low temperatures the rapid completion of the $\mathrm{H}$-bond network hinders molecular motions rather strongly. Ionic solutes and/or hydrostatic pressure disturb the $\mathrm{H}$-bond network and cause an increase in molecular mobility. This is fairly well demonstrated in Fig. 1 which compares the pressure and concentration dependence of a low temperature ${ }^{2} \mathrm{H}-T_{1}$ isotherm of $\mathrm{NaCl}$ and $\mathrm{KCl}$ dissolved in $\mathrm{D}_{2} \mathrm{O}[14,15]$.

Increasing pressure causes a dramatic increase in the rotational mobility $\left(\sim T_{1}\left({ }^{2} \mathrm{H}\right)\right)$ in neat water [17]. With dissolved ions the effect becomes less pronounced, and a qualitatively different behaviour emerges. Weakly hydrating ions like $\mathrm{K}^{+}, \mathrm{Cs}^{+}, \mathrm{I}^{-}$always further increase the rotational mobility by simply disrupting the $\mathrm{H}$-bond network without interacting strongly with water molecules. More strongly hydrating ions like $\mathrm{Na}^{+}, \mathrm{Li}^{+}, \mathrm{F}^{-}$show a twofold behaviour. At low hydrostatic pressure the $\mathrm{H}$-bond network is well developed and all simple ions, whether strongly or weakly hydrating, disrupt the network and increase the molecular mobility. This effect reaches a maximum at a concentration where only water molecules hydrating the ions are left. Often this concentration corresponds to the edge of the glassforming composition range. It is interesting to note that the concentration dependence of experimental glass transition temperatures $T_{\mathrm{g}}$ shows a minimum [18] also indicating an initially increasing molecular mobility upon addition of salt to water at low temperatures.

If, however, the H-bond network is already sufficiently disturbed ion - water interactions dominate over $\mathrm{H}$-bonding interactions and strongly hydrating ions cause a continuous slowing down of the rotational mobility of water molecules. In all solutions of alkali and alkali earth halides investigated cations exert a much more pronounced influence on the average mobility of water molecules than do anions (except for $\mathrm{F}^{-}$). This is convincingly demonstrated in Fig. 2.

With a strongly disturbed $\mathrm{H}$-bond network (high pressure or high solute concentration) very low temperatures can be reached. Then relaxation rates may be followed beyond their maximum $(\omega \tau \simeq 1)$ into the slow motion regime $(\omega \tau>1)$ as is demonstrated in Fig. 3. These relaxation rates have been interpreted with the simple motional model discussed.

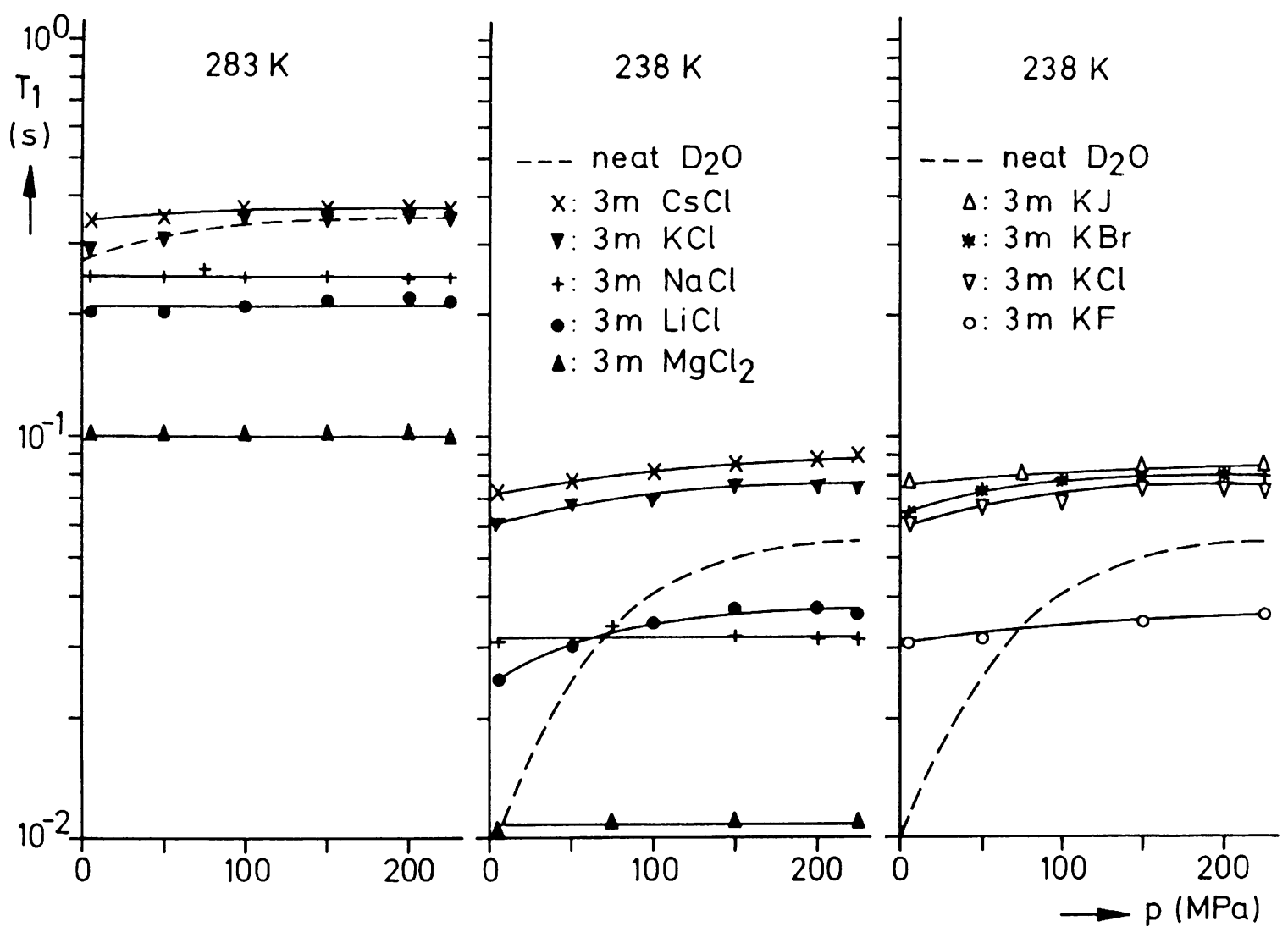

Fig. 2

Comparison of the effect of cations and anions on the average rotational mobility of water molecules in metastable ionic solutions 
The two unknown model parameters have been adjusted to the data of a concentrated solution preferably corresponding to a composition with only hydration water being present. Figure 4 illustrates the contribution from both the hydration and bulk site relaxation rates to the total rate in a 3 molal $\mathrm{NaCl} / \mathrm{D}_{2} \mathrm{O}$ solution.

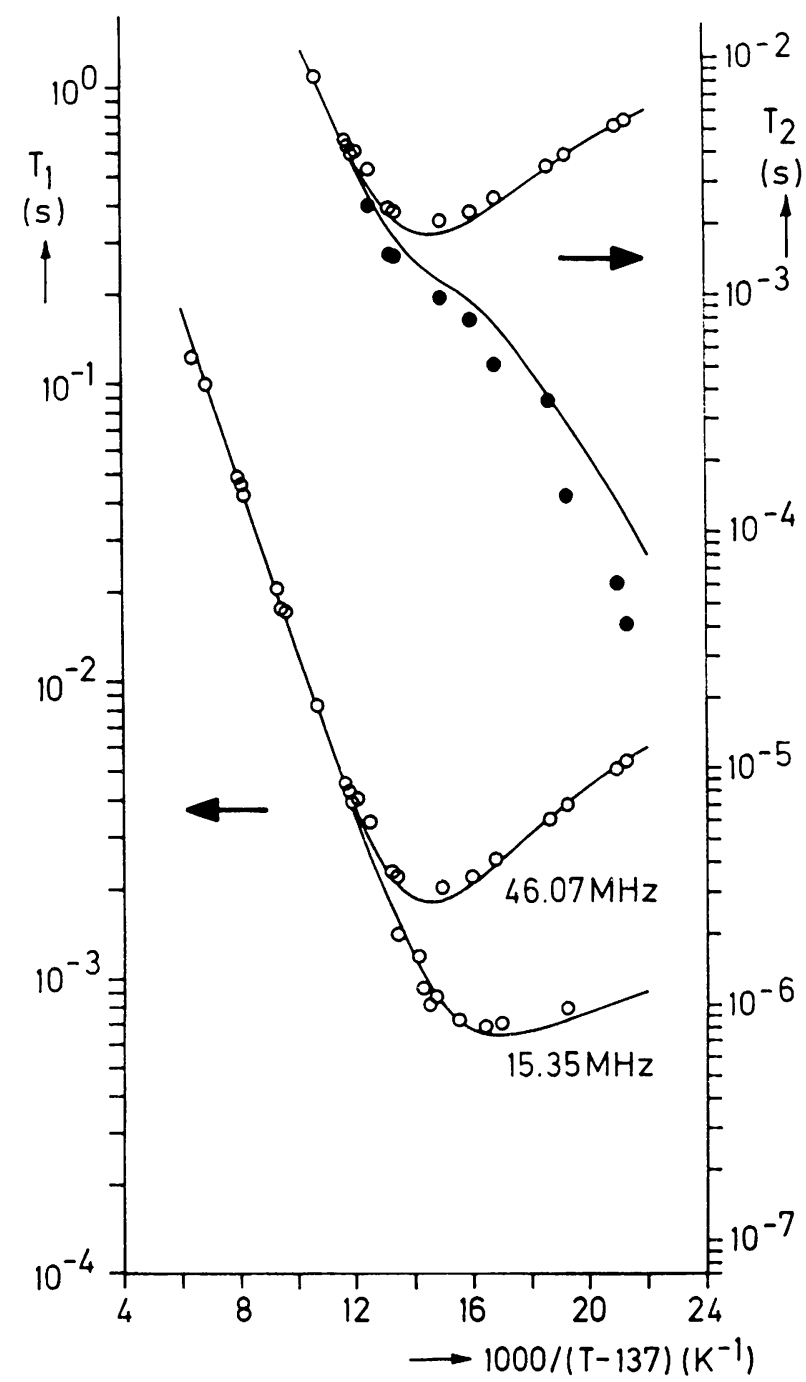

Fig. 3

Deuterium relaxation times in the fast and slow motions regimes in a modified Arrhenius diagram illustrated with a metastable $11 \mathrm{~m}$ $\mathrm{LiCl} / \mathrm{D}_{2} \mathrm{O}$ solution. $T_{0}=137 \mathrm{~K}$ represents the ideal glass transition temperature of the solution

The ideal glass transition temperatures $T_{0}$ deduced from these investigations are compiled in Fig. 5. An increase in $T_{0}$ upon addition of salt reflects a slowing down of cooperative tumbling modes of water molecules in these solutions, whereas a decreasing $T_{0}$ indicates increasing molecular mobility. Hence, in a strongly distorted $\mathrm{H}$-bond network with weak $\mathrm{H}$-bonding interactions strongly hydrating ions slow down molecular tumbling motions whereas weakly hydrating ions facilitate diffusional processes as do all simple dissolved ions in a well developed $\mathrm{H}$-bond network. Again cations exert a much stronger effect upon the glass transition temperature than do anions except for the $\mathrm{F}^{-}$-anion. These results suggest, that the influence of ionic solutes upon the

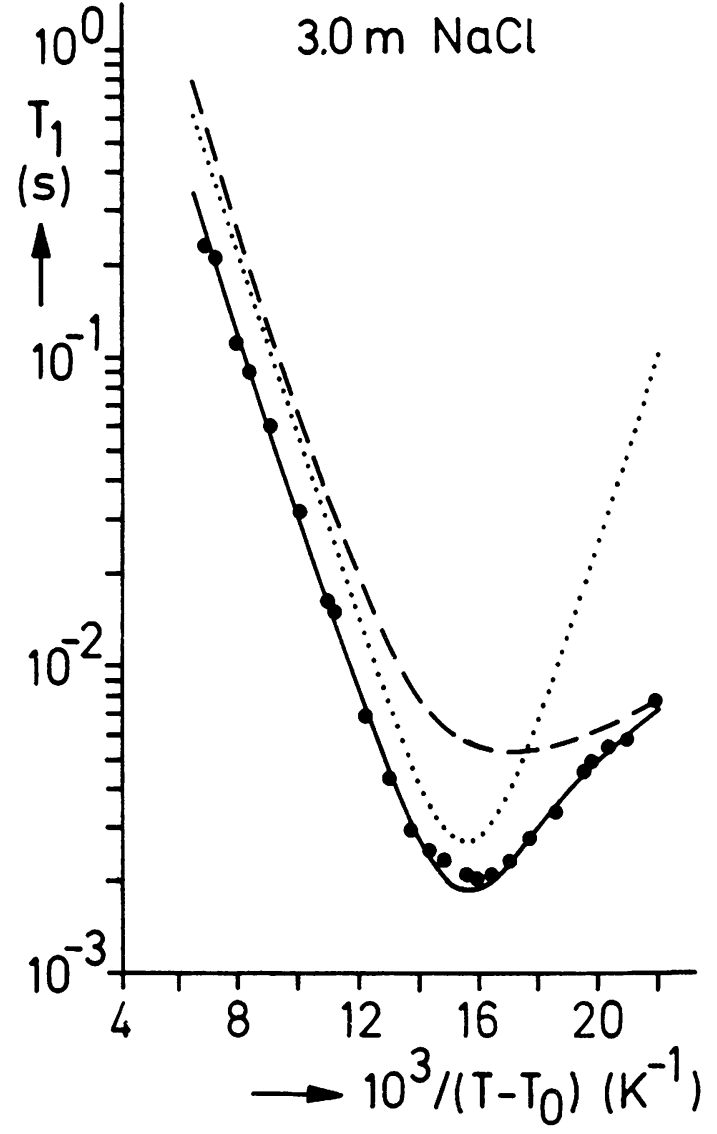

Fig. 4

The contribution from the cationic hydration water and the bulk water sites to the total deuterium relaxation time illustrated with a metastable $3 \mathrm{~m} \mathrm{NaCl} / \mathrm{D}_{2} \mathrm{O}$ solution under high pressure $(p=225 \mathrm{MPa})$

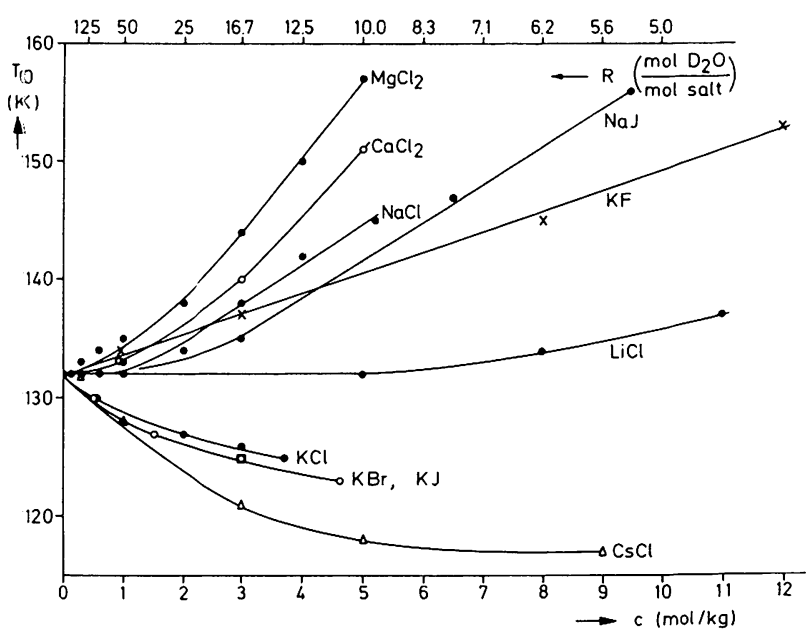

Fig. 5

Compilation of the ideal glass transition temperatures $T_{0}$ deduced from the deuterium relaxation rate curves obtained in metastable ionic solutions of concentration $c$ (molal)

temperature dependence of orientational and positional fluctuations is determined mainly by their effect on $T_{0}$.

\subsection{Undercooled Aqueous KF Solutions}

The $\mathrm{F}^{-}$-anion is of interest because of the orientation of water molecules relative to the anion in the hydration shell. 
Computer simulations and neutron scattering results concerning the $\mathrm{Cl}^{-}$coordination favour a linear configuration, whereas recent NMR data propose a symmetric arrangement $[19-22]$.

Besides the proton $\left({ }^{1} \mathrm{H}\right)$ and deuteron $\left({ }^{2} \mathrm{H}\right)$ relaxation, the ${ }^{19} \mathrm{~F}$ relaxation can also be studied conveniently in this system. Proton and fluorine nuclei relax via magnetic dipoledipole interactions. Their relaxation rate thus monitors reorientational and translational diffusive motions. Within the coordination sphere of the $\mathrm{F}^{-}$-anion ${ }^{19} \mathrm{~F}-{ }^{1} \mathrm{H}$ dipolar couplings are modulated by reorientational fluctuations of the water molecules. The corresponding relaxation rates depend sensitively on the distance between the interacting nuclei hence on the mean orientation of the water molecules in the hydration sphere. They have been calculated according to the simple motional model discussed $[23,24]$. The pertinent model parameters have been deduced from the deuterium relaxation suitably scaled according to the dynamic isotope effect. Positional fluctuations of water molecules have been accounted for within a force-free translational diffusion model with reflecting boundary conditions. The diffusion coefficients of ${ }^{1} \mathrm{H}$ and ${ }^{19} \mathrm{~F}$ are known at ambient temperature and pressure only [19]. They have been assumed to follow a VTF equation also. The distance of closest approach have been taken from radial distribution functions calculated with molecular dynamics techniques [21,22]. As an illustration Fig. 6 shows a comparison of the proton $R_{1}\left({ }^{1} \mathrm{H}\right)$ and fluorine $R_{1}\left({ }^{19} \mathrm{~F}\right)$ relaxation rate curves calculated with a linear and a symmetric anion - water arrangement respectively. The results clearly favour a linear configuration, at least in solutions with a strongly distorted $\mathrm{H}$-bond network.

\subsection{Undercooled $\mathrm{LiCl}$ Solutions}

Close to their respective glass transition regimes, dense metastable liquids often exhibit a nonexponential relaxation of structural fluctuations emerging either from a hierarchy of relaxation processes or from a superposition of exponentially relaxing processes implying many parallel relaxation paths.

Aqueous $\mathrm{LiCl}$ solutions provide the opportunity to study the dynamic behaviour of undercooled liquids which range from very difficult to vitrify to excellent glass formers by increasing the solute concentration $[2,4,16]$. In an 11 molal solution all water molecules may be considered to belong to the hydration sphere of the $\mathrm{Li}^{+}$cations. These solutions easily vitrify at low cooling rates.

Deuterium $\left({ }^{2} \mathrm{H}\right)$, proton $\left({ }^{1} \mathrm{H}\right)$ and lithium $\left({ }^{6} \mathrm{Li},{ }^{7} \mathrm{Li}\right)$ relaxation rates as well as ${ }^{1} \mathrm{H}$ and ${ }^{7} \mathrm{Li}$ self diffusion coefficients could be followed to very low temperatures close to the calorimetric glass transition temperatures $[2,4,16]$. With the model parameters adjusted to the ${ }^{2} \mathrm{H}$ relaxation rates, the corresponding rates of the protons $\left({ }^{1} \mathrm{H}\right)$ and lithium $\left({ }^{6} \mathrm{Li}\right)$ obtained at the same field ( $B_{0}=7.05$ Tesla) could be reproduced well $[5,16]$. This is shown in Fig. 7 and demonstrates the degree of consistency that could be obtained. Interpreting these data, exponentially decaying time correlation functions of the fluctuating variables have been assumed. However, relaxation data sampled at another field $\left(B_{0}=2.35\right.$ Tesla) clearly demonstrate deviations from the $\omega^{-2}$-Debye dependence of the corresponding spectral density functions in the case of the proton relaxation rates [24]. ${ }^{6} \mathrm{Li}$ and ${ }^{2} \mathrm{H}$ relaxation rate curves show no signs of nonexponential relaxations. The proton relaxation rates (together
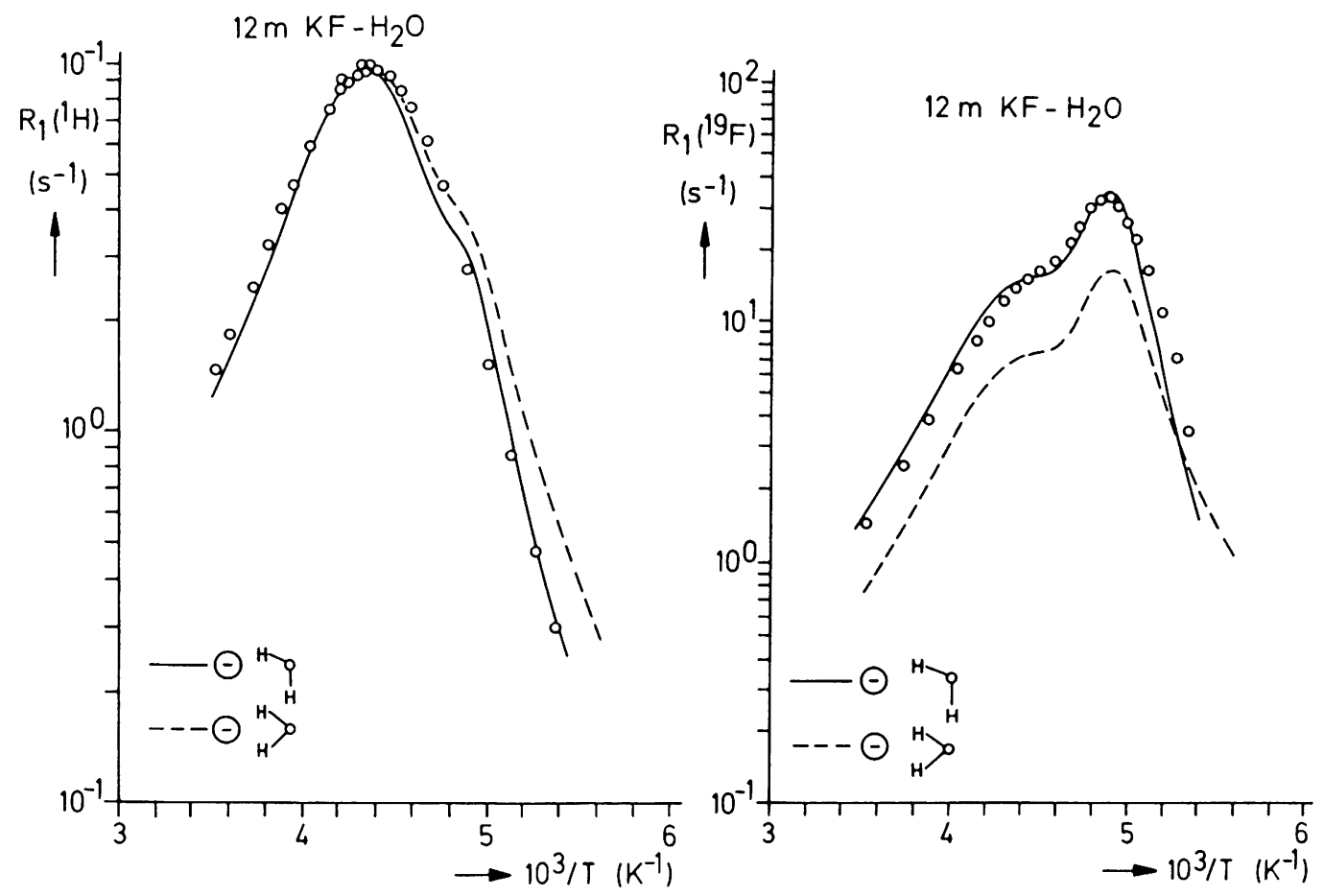

Fig. 6

Comparison of the ${ }^{1} \mathrm{H}$ and ${ }^{19} \mathrm{~F}$ spin-lattice relaxation rate curves in a metastable $12 \mathrm{~m} \mathrm{KF}$ solution calculated according to an almost linear ( $\longrightarrow$ ) and a symmetric (- --$)$ anion-water configuration 


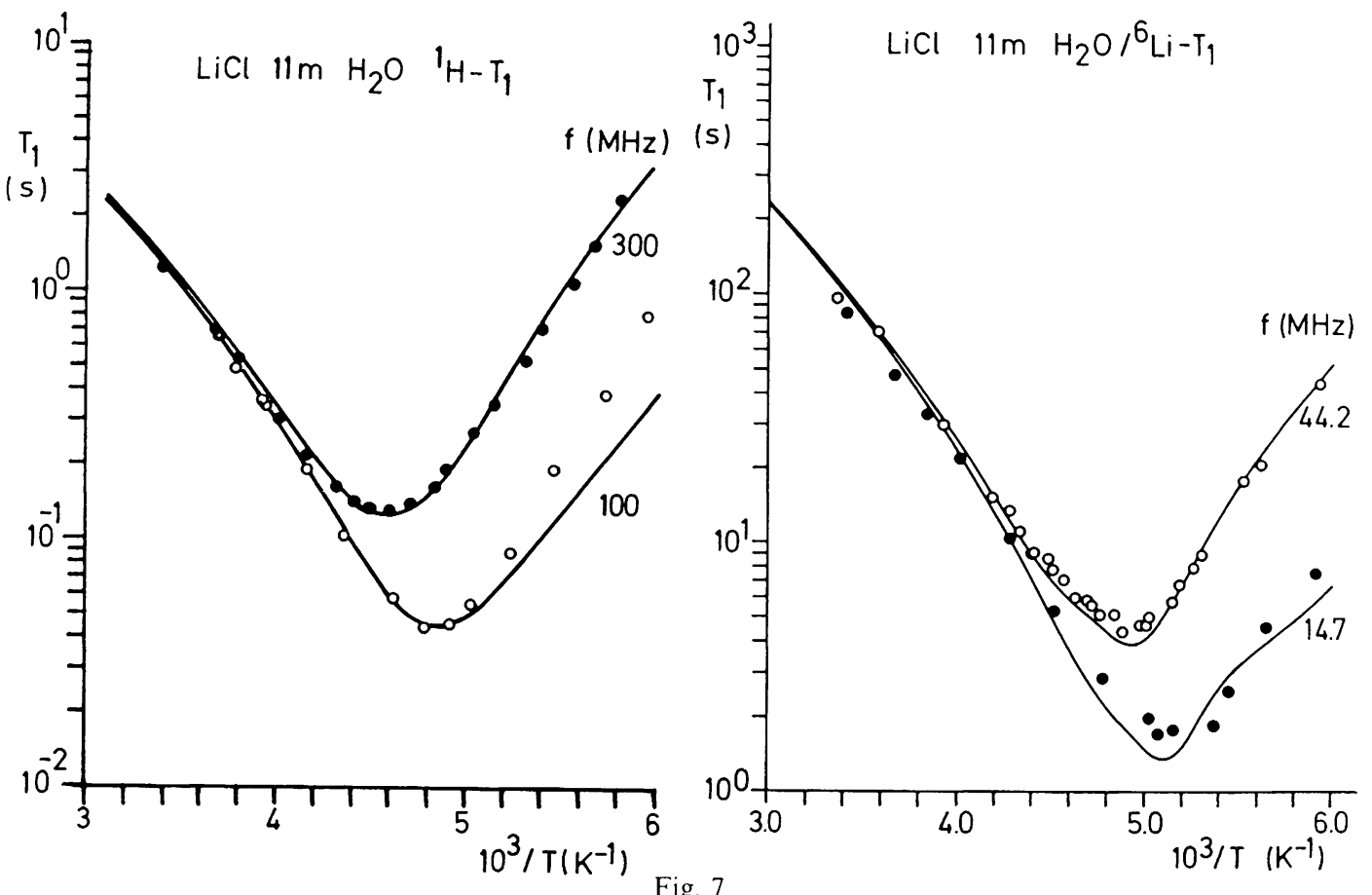

Comparison of calculated and experimental ${ }^{\prime} \mathrm{H}$ and " $\mathrm{Li}$ spin-lattice relaxation time curves in a $11 \mathrm{~m} \mathrm{LiCl}$ solution. Experiments have been performed at two magnetic fields $\left(B_{0}=7\right.$ Tesla, upper curve and $B_{0}=2.4$ Tesla, lower curve). Relaxation time curves have been calculated according to the two-mode approximation with exponential time correlation functions

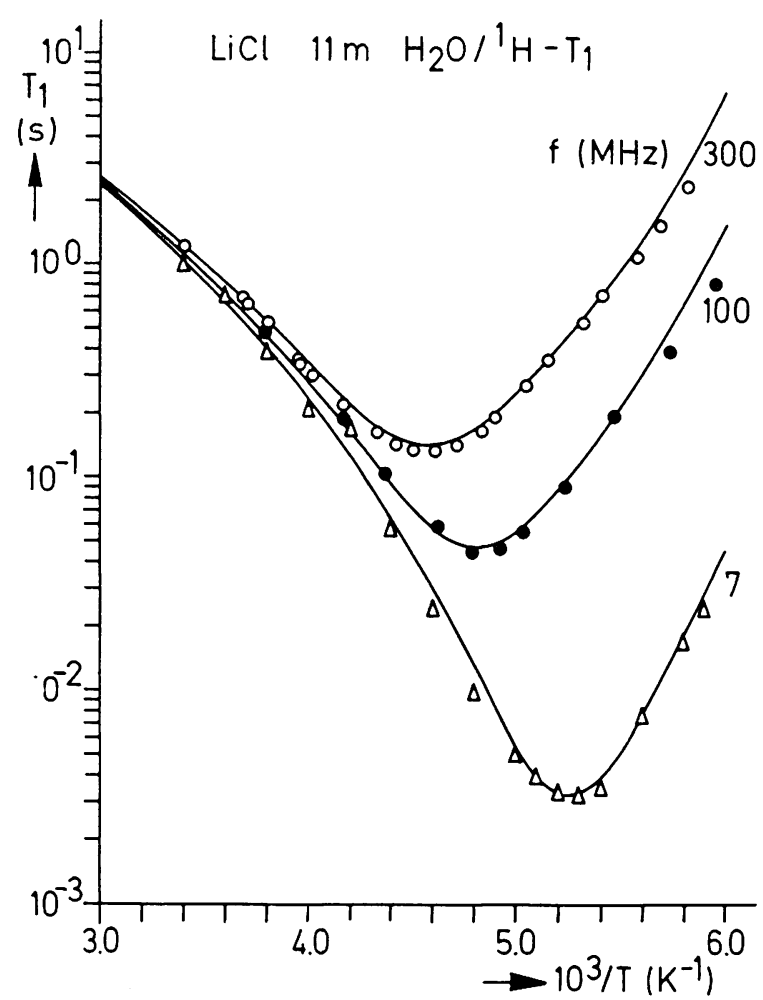

Fig. 8

Proton spin-lattice relaxation time curves of metastable $11 \mathrm{~m} \mathrm{LiCl}$ solutions obtained at different Larmor frequencies $(300 \mathrm{MHz}$, $100 \mathrm{MHz}, 7 \mathrm{MHz}$ ). Full curves correspond to a Cole-Davidson distribution of correlation times $\left(\beta_{\mathrm{CD}}=0.300\right)$ or, equivalently, to a stretched exponential time correlation function $\left(\beta_{\mathrm{KWw}}=0.435\right)$

with data taken from the literature) can be well represented, however, with a Cole-Davidson distribution of tumbling correlation times or, equivalently, with a Kohlrausch law [11] (stretched exponential) for the relevant time correlation function indicating the onset of nonexponential relaxation close to the glass transition temperature $T_{\mathrm{g}}$ (see Fig. 8). In this respect another recent investigation is of interest here [25]. Heitjans et al. studied the system $\mathrm{LiCl} / 7 \mathrm{D}_{2} \mathrm{O}$ with $\beta$-radiation detected NMR. The spin-lattice relaxation rate of ${ }^{8} \mathrm{Li}$ was measured to temperatures far below the glass transition temperature of the system. They could find indications for two dynamic processes and suggest that the local anisotropic modes are responsible for the weak relaxation rate maximum observed below the glass transition. Hence these local modes should be still activated in the glassy state.

\section{Conclusions}

NMR relaxation experiments on metastable aqueous solutions provide useful details about local structural features and characteristics of molecular motions hardly obtainable otherwise. A consistent interpretation of the $p, T, c, \omega$-dependence of transport - and relaxation coefficients of various species in these solutions places severe constraints on models of structure and dynamics. Hydrostatic pressure and/or ionic solutes serve to change the state of the random, transient $\mathrm{H}$-bond network in metastable water. Because of low thermal excitations the effect of $\mathrm{H}$-bonding and ionwater interactions upon structure and dynamics becomes especially pronounced and can be studied thoroughly.

We are grateful to Prof. Lüdemann for supporting this work and for many stimulating discussions. We also thank Prof. Heitjans for sending us a preprint of Ref. [28]. The skillful technical help of R. Knott, S. Heyn, and E. Treml made this work feasible.

\section{References}

[1] C. A. Angell in F. Franks ed. "Water-A Comprehensive Treatise", Vol. 7, p. 1-76, Plenum, New York 1982.

[2] C. A. Angell, E. J. Sare, J. Donnella, and D. R. MacFarlane, J. Phys. Chem. 85, $1461-64$ (1981). 
[3] H. G. Hertz, R. Tutsch, and H. Versmold, Ber. Bunsenges. Phys. Chem. 75, 1177-1191 (1971).

[4] E. W. Lang and H.-D. Lüdemann, Ber. Bunsenges. Phys. Chem. 89, 508-516 (1985).

[5] J. E. Enderby, in M.-C. Bellissant-Funel and G. W. Neilson eds., The Physics and Chemistry of Aqueous Ionic Solutions. Nato ASI Series C, Vol. 208, p. 129-145, Reidel, Dordrecht. Holland 1987.

[6] A. H. Narten, F. Vaslow, and H. A. Levy, J. Chem. Phys. 58 , 5017-5023 (1973).

[7] K. Heinzinger, Physica 13/B, 196-216 (1985)

[8] S. A. Rice and M. Sceats, J. Phys. Chem. 85, 1108-1119 (1981).

[9] E. W. Lang, H.-D. Lüdemann, and L. Piculell, J. Chem. Phys. 81. $3820-3827$ (1984)

[10] C. A. Angell, J. Phys. Chem. Solids 49, 863-871 (1988).

[11] J. Jäckle, Rep. Prog. Phys. 49, $171-231$ (1986).

[12] D. W. James and R. F. Armishaw, Aust. J. Chem. 28, $1179-1186(1975)$.

[13] W. Fink and E. W. Lang, J. Phys. Chem. 92, 6440-6445 (1988)

[14] W.Fink, H. Radkowitsch, and E. W. Lang, Chem. Phys. 124, $239-249(1988)$

[15] W. Fink, H. Radkowitsch, and E. W. Lang, Z. Naturforsch. $43 a, 538-546$ (1988)

[16] E. W. Lang and F. X. Prielmeier, Ber. Bunsenges. Phys. Chem. 92, 717-724 (1988)

[17] E. W. Lang and H.-D. Lüdemann, Angew. Chem. Int. Ed. Engl. 21, 315 (1982)
[18] K. Hofer in J. Dore and J. Teixeira eds. "Hydrogen Bonded Liquids", Nato ASI Series C, Reidel, 1989 in print.

[19] H. G. Hertz, G. Keller, and H. Versmold. Ber. Bunsenges. Phys. Chem. 73, 549-562 (1969).

[20] K. J. Müller and H. G. Hertz, Z. Phys. Chem. Neue Folge 140, $31-54$ (1984).

[21] R. Ahlrichs, H.-J. Böhm, H. G. Hertz, and K. J. Müller, Z. Phys. Chem. Neue Folge 142, 67-80 (1984).

[22] R. W. Impey, P. A. Madden, and I. R. McDonald, J. Phys Chem. 87, $5071-5083$ (1983).

[23] H. Radkowitsch and E. W. Lang, (1989) in preparation.

[24] E. W. Lang, W. Fink, and H. Radkowitsch in J. Dore and J. Teixeira, "Hydrogen Bonded Liquids", Nato ASI Series C. Reidel, 1989 in print.

[25] W. Faber. P. Heitjans. and A. Schirmer in D. Richter. A. J Dianoux, W. Petry, and J. Teixeira eds., "Dynamics of Disordered Materials", Springer Proceedings in Physics, Vol. 57 (1989).

Presented at the Joint Discussion Meeting of the Deutsche Bunsen-Gesellschaft für Physikalische Chemie, Associazione Italiana di Chimica Fisica, Faraday Division of the Royal Society of Chemistry, Société Française de Chimie, Division de Chimie Physique, "Transport Processes in Fluids and in Mobile Phases" Aachen, September 25 th to 27 th, 1989 